\title{
Management of atrial fibrillation: focus on the role of dronedarone
}

This article was published in the following Dove Press journal:

Open Access Emergency Medicine

20 September 2011

Number of times this article has been viewed

\section{Judy WM Cheng ${ }^{1,2}$}

'Massachusetts College of Pharmacy and Health Sciences, Boston, MA, USA; ${ }^{2}$ Brigham and Women's Hospital, Boston, MA, USA
Correspondence: Judy WM Cheng Massachusetts College of Pharmacy and Health Sciences, 179 Longwood Avenue, Boston, MA 02II5-5896, USA

$\mathrm{Tel}+\mathrm{I} 6177322868$

Fax + I 6177322244

Email judy.cheng@mcphs.edu
Background: Dronedarone is an amiodarone derivative that was approved in the US in July 2009 to reduce the risk of cardiovascular hospitalization in patients with paroxysmal or persistent atrial fibrillation (AF), who are in sinus rhythm (SR), or who will be cardioverted.

Objective: This article reviews the pharmacology, adverse effects, and clinical evidence available to date on the use of dronedarone in the management of AF and its potential role in the emergency department setting.

Results: In the EURIDIS and ADONIS studies evaluating the efficacy of dronedarone in maintaining SR, dronedarone significantly reduced the risk of recurrence of AF compared to placebo, by $22 \%$ and $27 \%$, respectively. The ERATO study examined the ability of dronedarone to control ventricular rate in permanent AF. The DIONYSOS study demonstrated that recurrences of $\mathrm{AF}$ were more frequent with dronedarone. However, discontinuation of therapy due to intolerance was more frequent with amiodarone. Furthermore, the ATHENA study demonstrated that dronedarone reduced mortality and cardiovascular hospitalization by $24 \%(P<0.05)$ in patients in SR but with other associated risks and a history of AF. However, the ANDROMEDA study, evaluating the use of dronedarone in patients with recent decompensated heart failure, and the PALLAS study, evaluating the use of dronedarone in patients with chronic AF, were both terminated prematurely due to a trend toward an increased risk of cardiovascular events.

Conclusion: Dronedarone has been demonstrated to be effective in reducing the incidence of AF recurrence. It appears to be less effective but better tolerated than amiodarone. Dronedarone appears to have a low proarrhythmic risk and is the first anti-arrhythmic that has been demonstrated to reduce cardiovascular mortality and cardiovascular hospitalization in clinically stable patients with other risk factors for recurrent AF. Therefore, dronedarone can be recommended as an anti-arrhythmic of choice in clinically stable patients for maintaining SR. If dronedarone is to be used in a patient with chronic stable heart failure, the patient must be monitored closely for any worsening of heart failure symptoms. The drug must be discontinued should the heart failure symptoms worsen.

Keywords: dronedarone, atrial fibrillation, anti-arrhythmics

\section{Introduction}

Atrial fibrillation (AF) is a common arrhythmia that brings patients to the emergency department and is associated with increased cardiovascular mortality, stroke, and hospitalization in the US. ${ }^{1}$ Risk of AF increases with age (incidence up to $26.0 \%$ for men and $23.0 \%$ for women by the age of 40$){ }^{2}$ At the emergency department, management of symptomatic AF includes rapid assessment of potential hemodynamic instability, the identification and treatment of the underlying or precipitating cause, and a careful 
assessment of the patient's history with particular attention to the risk of thromboembolism. At this time, management of AF involves achieving three major therapeutic goals: ventricular rate control (rate control); preventing thromboembolic events; and restoration of sinus rhythm (SR) (rhythm control). ${ }^{3}$ Rate control involves the use of atrioventricular nodal blocking agents such as beta-blockers, calcium channel blockers (diltiazem and verapamil) or digoxin. Patients who receive rate control management strategy will require longterm anticoagulation with warfarin or dabigatran therapy to prevent thromboembolic events. If they only have one of the following risk factors: congestive heart failure; hypertension; age $>75$ years; or diabetes, then aspirin may be used. Rhythm control involves the use of Class Ic and III anti-arrhythmic agents such as flecainide and amiodarone, for restoring and maintaining SR. Theoretically, one of the advantages of rhythm control therapy is that long-term anticoagulation may not be needed if the patient stays in SR after its restoration. ${ }^{3}$ However, studies evaluating rate vs rhythm control strategy indicate that long-term anticoagulation therapy may still be needed even if rhythm control strategy is used, as patients may still go back into AF and thromboembolic events may still occur. ${ }^{4}$ Numerous clinical trials have compared the rate control strategy with the rhythm control strategy with respect to long-term cardiovascular outcomes in patients with AF. From a therapeutic point of view, each strategy has its pros and cons. ${ }^{5}$ Overall, rate and rhythm control strategies are believed to produce non significant differences in longterm cardiovascular outcomes. The decision regarding the initial strategy of rate vs rhythm control in the emergency department depends on multiple factors including patient and physician preference, clarity of the history of onset of symptoms, type and duration of AF, severity of symptoms, patient age, and other associated cardiovascular diseases and medical conditions.

If rhythm control approach is chosen, cardioversion can be performed either electrically or pharmacologically. The pharmacologic rhythm control agents (ie, Class Ic and III anti-arrhythmics) that are currently available have variable efficacy and safety limitations. ${ }^{6}$ Amiodarone, dofetilide, ibutilide, flecainide, propafenone, procainamide, and quinidine have been studied and proven effective for pharmacologic cardioversion. ${ }^{3}$ Other agents including sotalol have been demonstrated to be effective for maintaining SR. ${ }^{3}$ Amiodarone is generally considered the agent with the best efficacy in restoring and maintaining SR., ${ }^{3,-10}$ In the Sotalol Amiodarone Atrial Fibrillation Efficacy Trial (SAFE-T), the largest trial of amiodarone in AF, amiodarone was shown to be significantly more efficacious than sotalol and placebo in maintaining SR (AF recurrence rate in 1 year: $48 \%$ for amiodarone, $68 \%$ for sotalol, and $87 \%$ for placebo, $P<0.001){ }^{8}$ In the Atrial Fibrillation Follow-up Investigation of Rhythm Management (AFFIRM) sub-study, amiodarone was also demonstrated to be the best agent in maintaining SR (AF recurrence rate in 1 year: $38 \%$ for amiodarone; $77 \%$ for other Class I agents; and $62 \%$ for sotalol, $P<0.001) .{ }^{10}$ Amiodarone is one of the few anti-arrhythmic agents that is well tolerated by patients with congestive heart failure and AF. ${ }^{11}$ In this patient population, amiodarone exerts minimal negative inotropic effects relative to other Class Ic and III anti-arrhythmic agents. ${ }^{12}$ In addition, amiodarone also demonstrates the lowest risk of Torsade de pointes among all Class Ic and III antiarrhythmic agents $(0.7 \%$ compared to $1.7 \%-4 \%$ for other agents). ${ }^{13}$ However, amiodarone does have an extensive side effect profile. Significant toxicities that require long-term monitoring include hypo- and hyperthyroidism, elevation of liver function enzymes, and pulmonary fibrosis. ${ }^{13}$ In addition, although amiodarone has been demonstrated to be efficacious in restoring and maintaining SR, it is not clear whether amiodarone has any meaningful impact on the long-term outcome of AF patients in terms of cardiovascular morbidity and mortality.

Dronedarone (Multaq ${ }^{\circledR}$, Sanofi Aventis, Paris, France) is a noniodinated benzofuran derivative of amiodarone that was approved by the Food and Drug Administration (FDA) in July 2009 for reduction of risk of cardiovascular hospitalization in patients with paroxysmal or persistent AF, who have had a recent episode of AF and have associated cardiovascular risk factors (including age $>70$, left ventricular ejection fraction $<40 \%$, hypertension, diabetes, and/or prior cerebrovascular accident) who are in SR or who will be cardioverted. ${ }^{14}$ Compared to amiodarone, dronedarone has shown a shorter half-life, decreased lipophilicity, and minimal noncardiovascular toxicity in clinical trials. The most recent update of the American Heart Association and American College of Cardiology Guidelines on the management of AF recommends the use of dronedarone as one of the first choices for rhythm control in patients with AF with no heart disease, hypertension without left ventricular hypertrophy, and coronary artery disease. ${ }^{3}$ This article reviews the pharmacology, adverse effects, and clinical evidence available to date on the use of dronedarone in the management of AF. Its role in the emergency department setting will also be discussed. 


\section{Clinical pharmacology \\ Mechanism of action}

Dronedarone (methanesulfonamide, N-(2-butyl-3[43\{(dibutylamino) propoxy\}benzoyl]-5-benzofuranyl)-, monochloride) has anti-arrhythmic properties similar to amiodarone. It possesses activity in all four Vaughan Williams classes. It has demonstrated rate-dependent inhibition of the rapid $\mathrm{Na}^{+}$current (Class I), $\alpha$ - and $\beta$-adrenergic receptor inhibition (Class II), blockade of $\mathrm{K}^{+}$outward currents as the main mechanism of action (Class III), and blockade of slow $\mathrm{Ca}^{2+}$ inward currents (Class IV). ${ }^{15,16}$ Class I and III effects increase refractory periods and decelerate cardiac conduction, providing mechanisms that induce rhythm control. Balanced inhibition of multiple outward currents may explain the decrease in the transmural dispersion of repolarization, which prevents significant proarrhythmic effects. ${ }^{17}$ Furthermore, in contrast to pure potassium channel blockers, dronedarone increases action potential duration and effective refractory period without reverse use-dependency, preventing the risk of early afterdepolarization. ${ }^{17,18}$ In addition, Class II and IV effects contribute to rate control properties in addition to the antiadrenergic (Class II) and blood pressure lowering (Class IV) effects of the drug. ${ }^{19,20}$ Table 1 summarizes the clinical pharmacologic profiles of amiodarone and dronedarone.

\section{Pharmacokinetics}

\section{Healthy volunteers}

Studies evaluating the pharmacokinetic profile of dronedarone in healthy volunteers and atrial fibrillation have not been published to date. The following information is obtained from the briefing document submitted by the manufacturer, Sanofi Aventis, to the FDA, as part of the supporting document for approval purposes. ${ }^{14}$ After oral administration in nonfasting conditions in healthy subjects, dronedarone is demonstrated to be at least $70 \%$ absorbed. However, its absolute bioavailability is only $15 \%$ due to significant first pass metabolism. Peak plasma concentrations of dronedarone are reached within 3 to 6 hours. At an oral dose of $400 \mathrm{mg}$ twice daily, steady state levels of the drug are reached within 4 to 8 days of treatment. Dronedarone was recommended to be taken with meals in all the efficacy/safety studies. It was reported that the impact of administration of dronedarone in the fasting state was significant (a 2-3 fold decrease in exposure) after a single dose. Such an impact would only be a $30 \%-40 \%$ decrease in exposure during chronic treatment.

Dronedarone is metabolized extensively by the cytochrome P450 (CYP) 3A4 enzyme into a main active metabolite,
SR35021, and numerous other nonactive metabolites. SR35021 contributes to $10 \%-30 \%$ of the pharmacological activity of dronedarone. Dronedarone is a moderate inhibitor of CYP3A4, CYP2D6, and P-glycoprotein. Both dronedarone and SR35021 exhibit high and nonsaturable protein binding $(>98 \%)$ in human plasma, primarily to albumin. The numerous metabolites of dronedarone are mainly excreted in the feces. Renal excretion is a very minor route of elimination ( $6 \%$ of the dronedarone dose). The steady state terminal elimination half-life of dronedarone is approximately 30 hours and that of SR35021 approximately 25 hours. Dronedarone is completely eliminated from plasma within 2 weeks after the last dose of $400 \mathrm{mg}$ twice daily treatment. ${ }^{14}$

\section{Special populations}

The pharmacokinetics of dronedarone in patients with AF are similar to those in healthy subjects. ${ }^{14}$ On average, female patients have dronedarone exposures 1.3-fold higher compared with male patients. In patients with body weight $\leq 60 \mathrm{~kg}$, exposures are 1.4-fold higher compared with patients with body weight $60-100 \mathrm{~kg}$. In patients $\geq 65$ years or $\geq 75$ years of age, exposures are 1.2-fold and 1.4-fold higher compared with patients $<65$ years old. ${ }^{14}$ History of heart failure and renal function do not significantly influence the pharmacokinetics of dronedarone in patients. ${ }^{14}$ Moderate hepatic impairment (definition not specified) modifies the pharmacokinetics of dronedarone: the steady-state dronedarone exposure increases by 1.3 -fold and active metabolite exposure decreases by $1.6-$ to 1.9 -fold. ${ }^{14}$ Safety and efficacy in children below the age of 18 years have not been established. ${ }^{21}$

\section{Pharmacodynamics/effect on electrocardiogram}

The effect of dronedarone on 12-lead ECG parameters (heart rate, PR, and QTc) was investigated in healthy subjects following repeated oral doses up to $1600 \mathrm{mg}$ once daily or $800 \mathrm{mg}$ twice daily for 14 days and $1600 \mathrm{mg}$ twice daily for 10 days. In the dronedarone $400 \mathrm{mg}$ twice daily group, there was no apparent effect on heart rate; a moderate heart rate lowering effect ( $\sim 4$ beats per minute) was noted at $800 \mathrm{mg}$ twice daily. There was a clear dose-dependent effect on PR-interval with an increase of $+5 \mathrm{~ms}$ at $400 \mathrm{mg}$ twice daily and up to $+50 \mathrm{~ms}$ at $1600 \mathrm{mg}$ twice daily. There was a moderate dose-related effect on the QTc-interval with an increase of $+10 \mathrm{~ms}$ at $400 \mathrm{mg}$ twice daily and up to $+25 \mathrm{~ms}$ with $1600 \mathrm{mg}$ twice daily. ${ }^{21}$ 
Table I Clinical pharmacologic profile of amiodarone vs dronedarone 21,37 $^{2}$

\begin{tabular}{|c|c|c|}
\hline & Dronedarone & Amiodarone \\
\hline $\begin{array}{l}\text { Vaughan Williams } \\
\text { Class }\end{array}$ & $\begin{array}{l}\text { All four classes, but the contribution of each of these activities } \\
\text { to the clinical effect is unknown }\end{array}$ & All four classes, but predominantly Class III \\
\hline Indications & $\begin{array}{l}\text { Reduction of risk of cardiovascular hospitalization } \\
\text { in paroxysmal/persistent AF/AFL with recent episode of AF/AFL } \\
\text { and associated cardiovascular risk factors }\end{array}$ & $\begin{array}{l}\text { Recurrent ventricular fibrillation; recurrent } \\
\text { hemodynamically unstable ventricular tachycardia; } \\
\text { supraventricular arrhythmias; acute management of AF; } \\
\text { long-term management in preventing recurrent AF }\end{array}$ \\
\hline Onset of action & 4-8 hours & $2-3$ days to $\mathrm{I}-3$ weeks \\
\hline Half-life & $13-19$ hours & $40-55$ days \\
\hline Protein binding & $>98 \%$ & $\sim 96 \%$ \\
\hline Metabolism & By CYP3A, CYP2D6 & By CYP3A4, CYP2C8 \\
\hline Route of elimination & $\sim 6 \%$ renal, $84 \%$ feces & Hepatic metabolism and biliary excretion \\
\hline Contraindications & $\begin{array}{l}\text { - Class IV heart failure or symptomatic heart failure } \\
\text { with a recent decompensation } \\
\text { - Second- or third-degree atrioventicular (AV) block or } \\
\text { sick sinus syndrome (except when used in conjunction with a } \\
\text { functioning pacemaker) } \\
\text { - Bradycardia }<50 \text { beats per minute } \\
\text { - Concomitant use of a strong CYP3A inhibitor } \\
\text { - Concomitant use of drugs or herbal products that } \\
\text { - Prolong the QT interval and may induce Torsade de pointes } \\
\text { - QTc Bazett interval } \geq 500 \text { ms } \\
\text { - Pregnancy } \\
\text { - Nursing mothers }\end{array}$ & $\begin{array}{l}\text { - Cardiogenic shock } \\
\text { - Severe sinus-node dysfunction, causing marked sinus } \\
\text { bradycardia; second- or third-degree atrioventricular } \\
\text { block; and when episodes of bradycardia have caused } \\
\text { syncope (except when used in conjunction } \\
\text { with a pacemaker) } \\
\text { - Hypersensitivity to the drug or to any of its } \\
\text { components, including iodine }\end{array}$ \\
\hline Precautions & $\begin{array}{l}\text { - Heart failure: If heart failure develops or worsens, consider } \\
\text { the suspension or discontinuation of therapy } \\
\text { - Liver injury: if hepatic injury is suspected, discontinue therapy } \\
\text { - Hypokalemia and hypomagnesemia: Maintain potassium and } \\
\text { magnesium levels within the normal range } \\
\text { - QT prolongation: Stop dronedarone if QTc Bazett } \geq 500 \text { ms } \\
\text { - Increase in creatinine: Within a week, dronedarone causes a } \\
\text { small increase in serum creatinine that does not reflect a } \\
\text { change in underlying renal function } \\
\text { - Teratogen: Women of childbearing potential should use } \\
\text { effective contraception while using dronedarone }\end{array}$ & 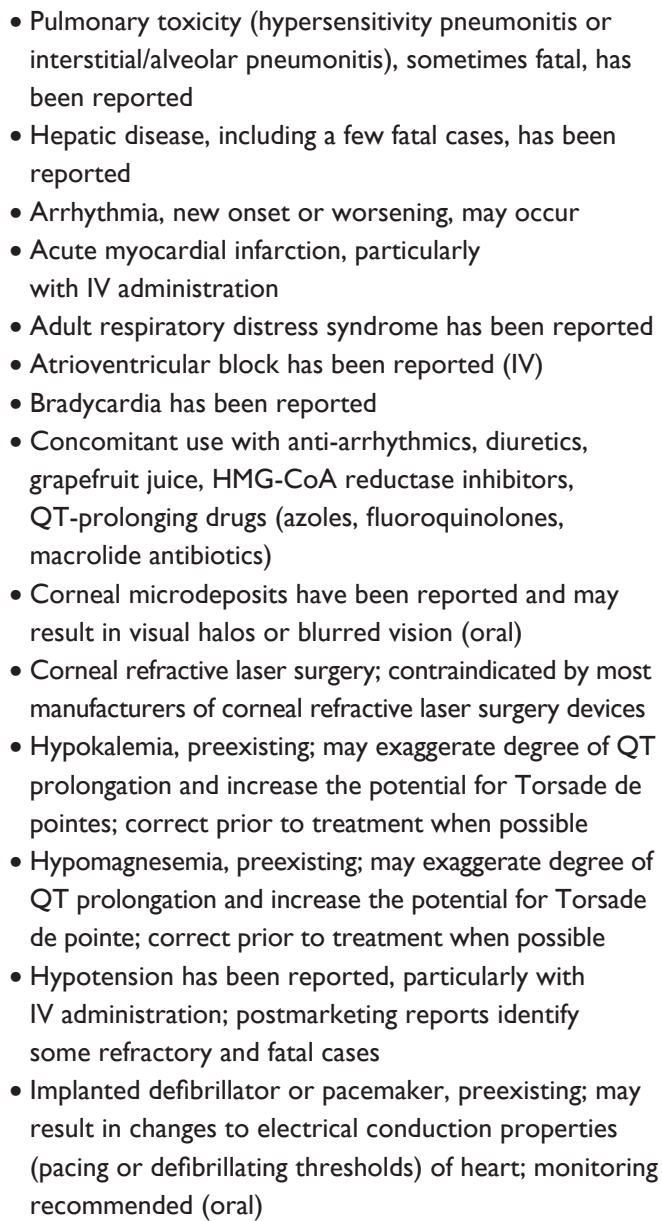 \\
\hline
\end{tabular}

(Continued) 
Table I (Continued)

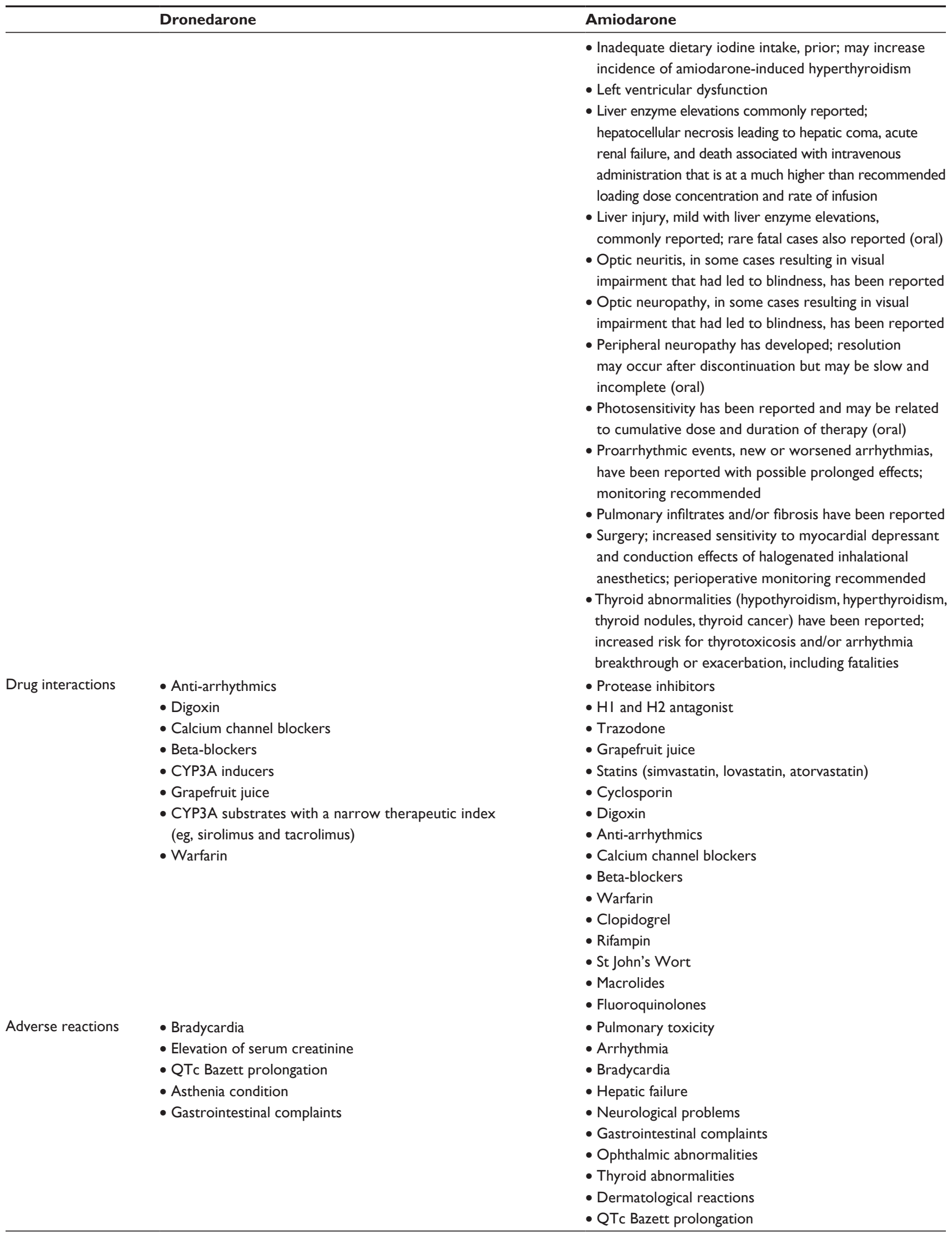

Abbreviations: AF, atrial fibrillation; $\mathrm{AFL}$, atrial flutter; $\mathrm{HF}$, heart failure. 


\section{Drug interactions}

Since a large portion of the clearance of dronedarone is mediated by CYP3A4, interaction of dronedarone and strong inhibitors of CYP3A4 has been studied. The administration of ketoconazole with a single $200 \mathrm{mg}$ dose of dronedarone has been reported to cause a 17 -fold increase in dronedarone exposure. The increase in exposure with ketoconazole was reported to be 5 - to 8 -fold with repeated $400 \mathrm{mg}$ twice daily doses of dronedarone. ${ }^{14}$ Moderate CYP3A4 inhibitors such as diltiazem, verapamil have been reported to have a modest effect (1.5-fold) on dronedarone exposures with no significant change on the active metabolite. ${ }^{14}$ Large consumption of grapefruit juice (double strength, large volume, three times daily), a CYP3A4 inhibitor, increases dronedarone exposure by 3 -fold. ${ }^{14}$ Strong CYP3A4 inducers such as rifampin have been reported to decrease dronedarone exposure by 5 -fold. ${ }^{14}$

Due to its inhibitory effect on CYP3A4, dronedarone $400 \mathrm{mg}$ twice daily has been reported to increase simvastatin exposure by 4 -fold. ${ }^{14}$ Dronedarone $400 \mathrm{mg}$ twice daily has also been reported to increase the exposure of verapamil by 1.4-fold, and nisoldipine by 1.5 -fold. ${ }^{14}$

Dronedarone $400 \mathrm{mg}$ twice daily has been reported to increase digoxin exposure by 2.5 -fold via its inhibitory action on the P-glycoprotein transporter. ${ }^{14}$ Exposure to dabigatran is also higher when it is administered with dronedarone than when it is administered alone (1.7- to 2-fold). ${ }^{14}$

Dronedarone is also a moderate inhibitor of CYP2D6. The interaction between dronedarone and metoprolol has also been studied. Forty-four healthy male subjects genotyped for CYP2D6 (39 extensive metabolizers, five poor metabolizers) were randomized to receive placebo $(n=12), 800 \mathrm{mg}(n=6)$, $1200 \mathrm{mg}(n=9)$, or $1600 \mathrm{mg}(n=17)$ of dronedarone daily after having taken metoprolol $200 \mathrm{mg}$ daily for 5 days. Metoprolol and dronedarone therapy were continued for an additional 8 days. ${ }^{22}$ Pharmacokinetic parameters of metoprolol were investigated at day 5 and at day 13. $C_{\max }$ and $A U C_{0-24 \mathrm{~h}}$ of metoprolol increased from days 5 to 13 in proportion to dronedarone dose only in CYP2D6 extensive metabolizer subjects $(P<0.001)$. Plasma metoprolol concentrations were highest in poor metabolizer subjects. Addition of dronedarone (800-1600 mg daily) to metoprolol (200 mg daily) increased bioavailability of metoprolol in CYP2D6 extensive metabolizers. Nevertheless at $400 \mathrm{mg}$ twice daily, (the approved therapeutic dose) these effects were modest.

\section{Clinical trials}

Pertinent clinical trials evaluating the clinical efficacy and safety of dronedarone are summarized in Table 2.
The Dronedarone for the Prevention of Atrial Fibrillation: a Dose-Ranging Study (DAFNE) study was a double-blind, randomized placebo-controlled, dose-ranging study that compared three different doses of dronedarone $(400 \mathrm{mg}$ twice daily, $600 \mathrm{mg}$ twice daily, and $800 \mathrm{mg}$ twice daily) with placebo for the maintenance of SR following electrical cardioversion in 270 patients with AF, treated for 6 months. ${ }^{23}$ The primary endpoint was time to first AF relapse over 6 months. There was an increase in time to AF recurrence with dronedarone $400 \mathrm{mg}$ twice daily compared to placebo (median 60 vs 5.3 days, relative risk reduction 55\%, 95\% confidence interval [CI]: $28 \%-72 \% ; P=0.001)$. There was no improvement in efficacy at the higher doses, although drug discontinuations occurred more frequently $(22.6 \%$ vs $3.9 \%$ on the $1600 \mathrm{mg}$ and $800 \mathrm{mg}$ daily doses of dronedarone, respectively). No proarrhythmic effect was observed in the study. This study established that dronedarone given orally in $400 \mathrm{mg}$ twice daily regimen is efficacious and safe enough to continue to further clinical trials. Premature drug discontinuations were mainly due to gastrointestinal side effects. No evidence of thyroid, ocular, or pulmonary toxicity was found in this study.

The EURopean trial In atrial fibrillation or flutter patients receiving Dronedarone for the maintenance of SR (EURIDIS) and American Australian African trial with DronedarONe In atrial fibrillation or flutter for the maintenance of SR (ADONIS) trials were double-blind, randomized, placebocontrolled trials, identical in design, evaluating the effect of dronedarone ( $400 \mathrm{mg}$ twice daily) in maintaining normal SR in 615 and 629 patients with AF/atrial flutter, respectively. ${ }^{24}$ At 12 months, dronedarone reduced the risk of $\mathrm{AF} /$ atrial flutter recurrence by $22 \%$ in the EURIDIS trial $(P=0.01)$ and by $27.5 \%$ in the ADONIS trial $(P=0.002)$. A post hoc analysis showed that dronedarone was associated with $34 \%$ and $27 \%$ lower risk of the combined endpoint of all-cause hospitalization or death, in the EURIDIS and ADONIS trials, respectively (hazard ratio [HR]: $0.73,95 \%$ CI: $0.57-0.93$; $P=0.01)$. The rates of cardiac and noncardiac adverse effects with dronedarone were comparable with placebo. Elevated serum creatinine levels were observed more frequently in the dronedarone group than in the placebo group (2.4\% vs $0.2 \%, P=0.004)$. These results suggest that dronedarone may have utility as an anti-arrhythmic agent beyond maintenance of normal SR.

The Efficacy and Safety of Dronedarone for the Control of Ventricular Rate during Atrial Fibrillation (ERATO) trial was a double-blind, randomized, placebo-controlled trial to evaluate the efficacy of dronedarone $400 \mathrm{mg}$ twice daily in 


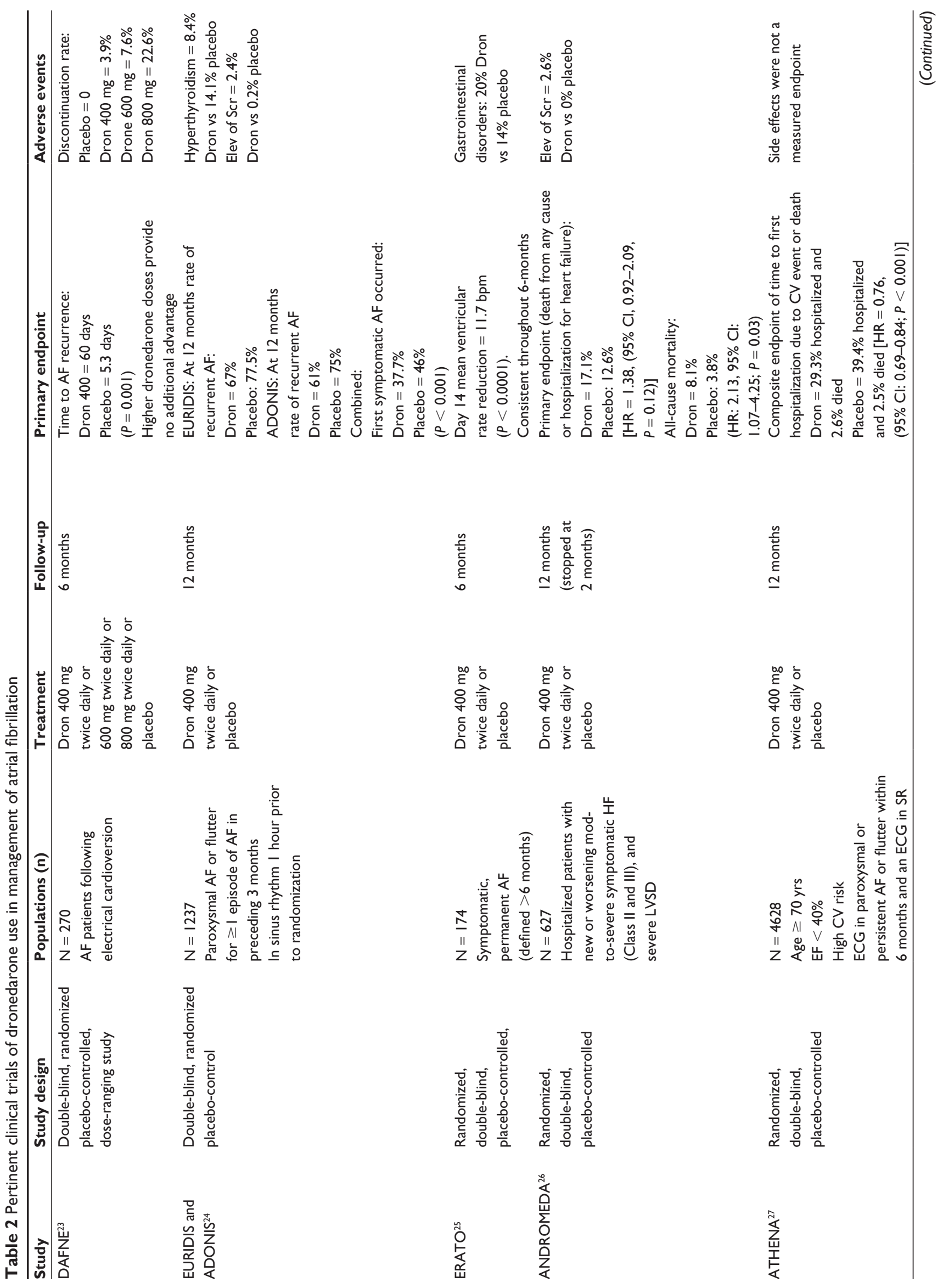




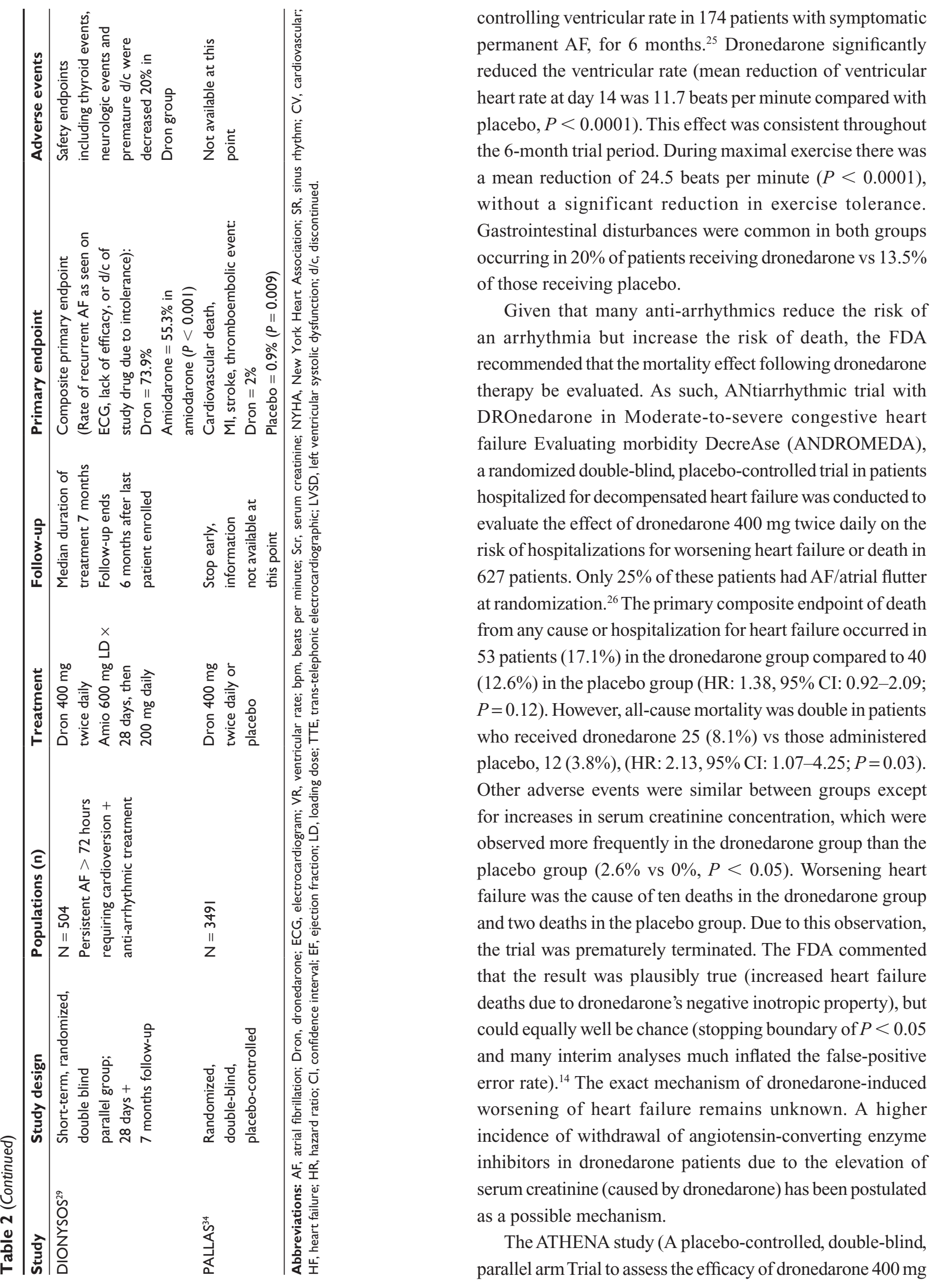


bid. for the prevention of cardiovascular Hospitalisation or death from any cause in patiENts with Atrial fibrillation/ atrial flutter) was conducted to further explore the effects of dronedarone on the risk of cardiovascular death and hospitalization. ATHENA was a randomized, double-blind, placebo-controlled trial where dronedarone $400 \mathrm{mg}$ twice daily was compared with placebo in addition to standard care among patients with a recent or current history of AF/atrial flutter. ${ }^{27}$ Based on the results of ANDROMEDA, patients with decompensated heart failure and Class III/IV heart failure were excluded. The primary outcome was a composite of first hospitalization due to cardiovascular events and all-cause mortality. Secondary outcomes were death from any cause, death from cardiovascular causes, and hospitalization due to cardiovascular events. A total of 4628 patients were randomized to either placebo or dronedarone. Treatment with dronedarone $400 \mathrm{mg}$ twice daily was associated with a $24 \%$ reduction of the combined risk of cardiovascular hospitalization or all-cause death (HR: $0.76,95 \%$ CI: $0.69-0.84$; $P<0.001)$ when compared with placebo. This reduction was driven by a $26 \%$ reduction in number of cardiovascular hospitalizations (HR: 0.74, 95\% CI: 0.67-0.82; $P<0.001$ ) and a $29 \%$ reduction in cardiovascular deaths (HR: 0.71 , 95\% CI: $0.51-0.98 ; P=0.03)$. The decrease in the number of cardiovascular hospitalizations with dronedarone was mainly due to a reduction in hospitalizations for AF (HR: $0.63,95 \%$ CI: $0.55-0.72 ; P<0.001)$. In addition, significantly fewer hospitalizations for an acute coronary syndrome were observed in the dronedarone group (HR: $0.70,95 \% \mathrm{CI}$ : $0.51-0.97 ; P=0.03)$. There were no significant differences between the two groups in the number of hospitalizations for heart failure, ventricular arrhythmia or nonfatal cardiac arrest, or noncardiovascular reasons. ${ }^{28}$ The dronedarone group had higher rates of bradycardia ( $3.5 \%$ vs $1.2 \%, P<0.001)$, QT interval prolongation $(1.7 \%$ vs $0.6 \%, P<0.001)$, nausea (5.3\% vs $3.1 \%, P<0.001)$, diarrhea $(9.7 \%$ vs $6.2 \%$, $P<0.001)$, rash (3.4\% vs $2.0 \%, P<0.006)$, and increased serum creatinine level $(4.7 \%$ vs $1.3 \%, P<0.001)$ than the placebo group. However, thyroid- and pulmonary-related adverse events were not significantly different between the two groups. This trial demonstrated a beneficial effect of dronedarone on cardiovascular morbidity and mortality. However, its place in therapy relative to amiodarone or other AF medications cannot be elucidated from these data. Although not the primary endpoints, a post hoc analysis of ATHENA also evaluated the rhythm- and rate-controlling properties of dronedarone. ${ }^{29}$ The median time to first AF or atrial flutter recurrence in patients in SR at baseline was
498 days in placebo patients and 737 days in dronedarone patients (HR: $0.749,95 \%$ CI: $0.68-0.82 ; P<0.001$ ). In the dronedarone group, 339 patients $(15 \%)$ had $>1$ electrical cardioversion, compared to 481 (21\%) in the placebo group (HR: $0.684,95 \%$ CI: $0.60-0.79 ; P<0.001$ ). The likelihood of permanent $\mathrm{AF}$ or atrial flutter was lower with dronedarone (178 patients [7.6\%]) compared to placebo (295 patients [12.8\%]; $P<0.001)$. At the time of first AF or atrial flutter recurrence, the mean heart rates were 85.3 and 95.5 beats/ minute in the dronedarone and placebo groups, respectively $(P<0.001)$. The investigators concluded that dronedarone demonstrated both rhythm- and rate-controlling properties in ATHENA. These effects are likely to contribute to the reduction of important clinical outcomes observed in this trial.

Although the ATHENA study was not supposed to enroll patients with Class III heart failure, there were 209 patients with New York Heart Association (NYHA) Class II/III congestive heart failure (CHF) and a left ventricular ejection fraction $\leq 40 \%$ (114 placebo, 95 dronedarone patients) enrolled. ${ }^{30} \mathrm{In}$ this subgroup of patients, a primary outcome event occurred in 59/114 placebo patients compared with 42/95 dronedarone patients (HR: $0.78,95 \%$ CI: 0.52-1.16). Twenty of 114 placebo patients and twelve of 95 dronedarone patients died during the study (HR: 0.71, 95\% CI: 0.34-1.44). Fifty-four placebo and 42 dronedarone patients were hospitalized for an intermittent episode of NYHA Class IV CHF (HR: 0.78, 95\% CI: $0.52-1.17)$. The investigators concluded that in patients with stable heart failure, dronedarone did not increase mortality and cardiovascular hospitalization. However, it is still important to note that should patients' heart failure symptoms worsen, dronedarone therapy should be withheld based on the result of the ANDROMEDA study.

The Efficacy and Safety of Dronedarone versus Amiodarone for the Maintenance of SR in Patients with Atrial Fibrillation (DIONYSOS) study, is a randomized, doubleblind trial that was designed to compare dronedarone $(400 \mathrm{mg}$ twice daily) and amiodarone (600 mg daily for 28 days and $200 \mathrm{mg}$ daily thereafter) in 504 patients with $\mathrm{AF}^{31}$ At 12 months, significantly more patients in the dronedarone group reached the primary endpoint defined as recurrence of AF or premature study drug discontinuation for intolerance or lack of efficacy compared to amiodarone ( $73.9 \%$ vs $55.3 \%$, $P<0.0001)$. Recurrences of AF were more frequent with dronedarone (63.5\% vs $42.0 \%, P=$ not reported), whereas premature study drug discontinuations due to intolerance were less frequent with dronedarone, although not significantly so, compared to the amiodarone group (10.4\% vs $13.3 \%, P=0.13)$. Patients on dronedarone compared with 
amiodarone had a nonsignificant decrease in the incidence of the predefined composite safety endpoint including thyroid, hepatic, pulmonary, neurological, skin, ocular, and gastrointestinal adverse events as well as premature study drug discontinuation due to any adverse event (83 vs 107 , $P=0.1291)$. In the dronedarone arm less thyroid events ( $2 \%$ vs $15 \%$ ), neurological events ( $3 \%$ vs $17 \%$ ) and premature study drug discontinuation due to any adverse events (13\% vs 28\%) were observed ( $P$ values not reported). In contrast, gastrointestinal events (diarrhea, vomiting, nausea) were more frequent in the dronedarone arm (32\% vs $13 \%)$. Pronounced QTc prolongation was experienced less often in the dronedarone arm than the amiodarone arm (27\% vs $52 \%)$ and no episodes of Torsade de pointes occurred throughout the study. Although the duration of this study was too short to develop definitive conclusions regarding long-term efficacy and safety of dronedarone directly compared to amiodarone, these data help identify the place in therapy for dronedarone. Dronedarone appears to improve tolerance at the cost of decreased efficacy.

A meta-analysis was performed based on eight randomized placebo-controlled trials of dronedarone and amiodarone. ${ }^{32}$ The analysis included studies that enrolled AF patients, had follow-up for greater than 6 months, and reported recurrent AF or all-cause mortality as endpoints. The number of dronedarone-treated patients was substantially higher than the number of amiodarone-treated patients (5967 vs 669). A significant reduction in recurrent AF compared to placebo was observed with amiodarone (odds ratio [OR]: 0.12, 95\% CI: $0.08-0.19$,) but not with dronedarone (OR: $0.79,95 \%$ CI: 0.33-1.87). The superiority of amiodarone over dronedarone for the prevention of recurrent AF was suggested in a logistic regression model (OR: 0.49, 95\% CI: 0.37-0.63; $P<0.001)$. However, the models also identify a trend toward greater all-cause mortality (OR: 1.61, 95\% CI: 0.97-2.68; $P=0.066)$ and greater overall adverse events requiring drug discontinuation (OR: 1.81, 95\% CI: 1.33-2.46; $P<0.001$ ) with amiodarone compared to dronedarone. Combining the results from this meta-analysis and the DIONYSOS study, the authors estimated that for every 1000 patients treated with dronedarone instead of amiodarone, there would be 228 more recurrences of AF at 1 year in exchange for 9.6 fewer deaths and 62 fewer adverse events requiring therapy discontinuation. Several limitations of the analysis may involve differences in patient populations. Permanent AF was excluded from all dronedarone studies, while paroxysmal AF was excluded from two of the four amiodarone trials. Only one (amiodarone) study included highly symptomatic AF patients while in clinical practice elimination of symptoms due to AF is often the primary reason to utilize the rhythm control strategy.

\section{Adverse events}

The mean dronedarone exposure across the studies was 12 months. ${ }^{23-31}$ In ATHENA, the mean and maximum follow-up was 21 months and 30 months, respectively. ${ }^{27}$ Of note, while the ATHENA study enrolled patients $\geq 70$ years of age $(n=4628)$, patients in other trials were relatively young (mean age $<66$ years) compared to the population at risk who will utilize dronedarone. ${ }^{23-31}$ In addition, no patients had severe congestive heart failure at baseline and almost all the patients had a left ventricular ejection fraction greater than $35 \% .{ }^{14,23-31}$ Overall, from all the clinical trials, the incidence of deaths was similar in the dronedarone (1.2\%) and placebo $(1.1 \%)$ groups during the study period. Dronedarone was associated with a higher risk of gastrointestinal disorders compared to placebo ( $1.9 \%$ vs $0.5 \%, P$ not reported $).{ }^{14}$ Other adverse events of dronedarone reported included diarrhea, nausea or vomiting, serum creatinine increase (shown to be related to inhibition of creatinine secretion at kidney tubular level without decrease in glomerular filtration), rash, and cardiac effects (bradycardia, QT prolongation).

Unlike amiodarone, dronedarone does not appear to significantly increase the risk of thyroid, neurological, hepatic, or pulmonary toxicity in clinical studies. ${ }^{14,23-31}$ However, longer term data will be required to confirm this. Recently (January 2011), the FDA has announced that several case reports of hepatocellular liver injury and hepatic failure in patients treated with dronedarone, including two post-marketing reports of acute hepatic failure requiring transplantation have been received. ${ }^{33}$ Because these reactions are reported voluntarily from a treatment population of unknown size, it was not possible to reliably estimate their frequency or establish a causal relationship to drug exposure. The two cases of acute hepatic failure requiring transplantation occurred at 4.5 and 6 months after initiation of dronedarone in patients with previously normal hepatic serum enzymes. Both patients were female and approximately 70 years of age. In the first case, the patient had underlying intermittent atrial fibrillation, arterial hypertension, and stable coronary artery disease. She was treated with dronedarone for 4.5 months. Two weeks prior to hospitalization she reported increased exhaustion and tiredness. One week prior to admission she discontinued dronedarone, and at the time of admission she was noted to have jaundice, coagulopathy, transaminitis, and hyperbilirubinemia, which progressed to 
hepatic encephalopathy over the next 9 days. A pre-transplant workup did not reveal another etiology of liver failure. In the second case, the patient had a medical history of paroxysmal atrial fibrillation and Sjögren's syndrome. Following 6 months of treatment with dronedarone she developed weakness, abdominal pain, coagulopathy, transaminitis, and hyperbilirubinemia. She was transplanted 1 month later; no alternative etiology for liver failure was identified in the transplant work-up. In both cases, the explanted liver showed evidence of extensive hepatocellular necrosis. Liver injury is now added as a warning in the official prescribing information of dronedarone. ${ }^{21}$

More recently, the PALLAS study examining the use of dronedarone compared to placebo in approximately 3000 patients with permanent $\mathrm{AF}$ ( $>2$ years), who were $>65$ years of age and had at least one additional risk factor including history of coronary artery disease, previous stroke, heart failure, or diabetes, was stopped prematurely due to a significant increase in cardiovascular events in the dronedarone arm (cardiovascular death, MI, stroke, thromboembolic event: dronedarone $2 \%$, placebo, $0.9 \%, P=0.009) .{ }^{34}$ Full details of this study are not currently available. However, it is a second indication (in addition to the ANDROMEDA study) that dronedarone may not be suitable for sicker patients.

\section{Precautions and contraindications}

Based on findings from clinical studies, dronedarone is contraindicated for use in the following populations: (1) Class IV heart failure or symptomatic heart failure with a recent decompensation (within last month); (2) second- or third-degree atrioventicular block or sick sinus syndrome (except when used in conjunction with a functioning pacemaker); (3) bradycardia $<50$ beats per minute; (4) concomitant use of a strong CYP3A inhibitor; (5) concomitant use of drugs or herbal products that prolong the QT interval and may induce Torsade de pointes; (6) severe hepatic impairment; (7) patients with QTc Bazett interval $\geq 500 \mathrm{~ms}$; and (8) pregnant or nursing women. ${ }^{21}$

Several precautions and warnings were also issued by the manufacturer. Symptoms of heart failure should be monitored during dronedarone therapy. If heart failure develops or worsens, clinicians should consider withholding or discontinuing dronedarone therapy. If liver injury is suspected during treatment, one should consider discontinuing or not re-initiating dronedarone. Clinicians should also be vigilant about monitoring potassium and magnesium serum concentrations and make sure that they are within the normal range to minimize risk of proarrhythmia. Dronedarone should be withheld if a patient's QT interval exceeds $500 \mathrm{~ms}$ at any time. ${ }^{21}$

\section{Dosage and administration}

The recommended dose of oral dronedarone is $400 \mathrm{mg}$ twice daily in adults. Adjustment of dosage in poor renal function is not necessary. There is no recommendation on dronedarone dosage adjustment in patients with liver function abnormalities. Based on a pharmacokinetics study, moderate liver dysfunction increases dronedarone exposure. ${ }^{14}$ Due to the recent report of possible dronedarone-induced liver injury, use of dronedarone in those patients should be avoided. Dronedarone therapy can be initiated in both inpatient and outpatient settings.

It is to be expected that, in a clinical situation, many patients who require dronedarone therapy will already be on other anti-arrhythmic agents. It is therefore important to consider a safe strategy in therapy switching in order to prevent adverse events, specifically additive effects in prolonged QT intervals and risk of Torsade de pointes. In the ANDROMEDA and ATHENA studies, patients who received prior amiodarone treatment, therapy was discontinued at least 4 weeks before randomization. ${ }^{26,27}$ Other Class I and III anti-arrhythmic agents were withdrawn for at least five plasma half-lives prior to the first dose of dronedarone administration (Pers comm, Sanofi-Aventis, 2009). In the ERATO study, patients' amiodarone therapy was stopped for at least 2 months prior to enrollment. ${ }^{14}$ Since ERATO enrolled patients with chronic AF on rate control therapy only, no patients were receiving other Class I and III anti-arrhythmic agents immediately prior to the study. In the EURIDIS and ADONIS trials, patients who were previously on amiodarone were allowed into the trial and dronedarone could be initiated immediately after discontinuation of amiodarone therapy. Patients who were receiving other Class I and III anti-arrhythmic agents at the time of screening were excluded from the study. ${ }^{24}$ No adverse events relating to therapy switching were reported in the EURIDIS and ADONIS trials.

As the recommendation for handling prior anti-arrhythmic agents varies among clinical trials, it is important to design the switching strategy based on individual patient characteristics and other comorbidities. For instance, for patients who are switching therapy because of intolerance to prior anti-arrhythmic agents, and whose risk of AF recurrence without anti-arrhythmics is not high, it is probably advisable to wait until the previous anti-arrhythmic agents are washed out of the system before initiating dronedarone. 
This means five plasma half-lives of the anti-arrhythmic agents. Except for amiodarone, this will be $\sim 2-3$ days. In patients who were on amiodarone previously, waiting for at least 4 weeks (if possible) would be advisable. Amiodarone has an average half-life of $\sim 45$ days, therefore theoretically, it will not be completely washed out for $\sim 225$ days. If patient's risk of AF recurrence is high, such as in those with heart failure, uncontrolled hypertension, AF longer than 3 months duration, age $>70$ years, left atrial enlargement, or rheumatic heart disease, ${ }^{3}$ a prolonged waiting period for the previous agent to be washed out may not be advisable. In these cases, if patients were on Class I and III anti-arrhythmic agents in addition to amiodarone, dronedarone can still be initiated after the previous agents have been stopped for 2-3 days. If patients were previously on amiodarone, dronedarone can probably be started immediately after amiodarone discontinuation (without waiting for 4 weeks) with more frequent ECG monitoring for prolonged QT intervals during the first week after initiation of dronedarone therapy (dronedarone terminal half-life is $\sim 30$ hours. It will take five half-lives [ 150 hours] to achieve maximum steady state serum concentration). The Optimal Timing of Dronedarone Initiation After Conversion in Patients With Persistent Atrial Fibrillation (ARTEMIS Load) study is currently ongoing to address this issue. ${ }^{35}$

\section{Future perspective and role in the emergency department}

Clinical studies performed to date indicate that dronedarone $400 \mathrm{mg}$ given orally twice daily is the optimal dose (greatest efficacy and least toxicity). Based on the results of currently available clinical trials, dronedarone is effective in reducing incidence of recurrence of AF in patients who have been cardioverted back to SR. Dronedarone is not a medication that is meant for acute conversion back to SR. Although it appears to be less effective yet better tolerated than amiodarone, dronedarone appears to have a low proarrhythmic risk. Only one case of Torsade de pointes was reported in all the clinical trials after 10 months of treatment. This patient also had other risk factors for proarrhythmia, including prolonged baseline QT interval and borderline low serum potassium concentration. ${ }^{14}$ Dronedarone is the first anti-arrhythmic that has been demonstrated to not only maintain SR, but to reduce cardiovascular morbidity and mortality in clinically stable patients with other risk factors for recurrent AF, including stable heart failure patients. Therefore, dronedarone can be recommended as an anti-arrhythmic of choice in clinically stable patients with associated risk factors for recurrent AF, including those with no heart disease, hypertension without left ventricular hypertrophy, and coronary artery disease. ${ }^{3}$ Amiodarone may be reserved as an alternate agent in patients failing on dronedarone (ie, with recurrent $\mathrm{AF}$ ) if it is determined that it is essential to maintain the patient in SR. However, if dronedarone is to be used in a patient with chronic stable heart failure, the patient must be monitored closely for any worsening of heart failure symptoms. Patients presenting to the emergency department on dronedarone should be evaluated for possible worsening of signs and symptoms of heart failure, and questioned about recent hospitalization due to heart failure. Should the patient demonstrate worsening of symptoms of heart failure, or have documented recent hospitalization for heart failure, dronedarone therapy should be discontinued and an alternative agent, such as amiodarone, should be considered.

Dronedarone has not been compared with other commonly prescribed anti-arrhythmic agents such as sotalol or dofetilide for management of AF in terms of efficacy or tolerability. However, a mixed-treatment comparison model study of 39 randomized double-blind placebo control studies of different anti-arrhythmics (including amiodarone, dronedarone, sotalol, felcainide, and propafenone) demonstrated that amiodarone had the largest effect in reducing AF recurrence (OR: 0.22, 95\% CI: 0.16-0.29) but was associated with the highest rate of patients experiencing at least one serious adverse event (OR: 2.41, 95\% CI: 0.96-6.06) and treatment withdrawals due to adverse events (OR: 2.91, 95\% CI: 1.66-5.11). Dronedarone was associated with the lowest rate of proarrhythmic events including bradycardia (OR: 1.45, 95\% CI: 1.02-2.08) compared to all other agents. ${ }^{36}$

Unlike amiodarone, dronedarone has not been studied for management of other arrhythmias such as ventricular arrhythmia, another common arrhythmia in heart failure patients. Future clinical studies may want to explore these aspects. Dronedarone has also not been studied for other types of supraventricular arrhythmia. In addition, numerous clinical trials in recent years comparing rate control strategy vs rhythm control strategy have demonstrated that both treatment approaches are similar in terms of long-term cardiovascular outcomes in patients with AF, including heart failure patients, who were traditionally believed to be better served by maintaining SR from a symptom control perspective. ${ }^{5}$ Dronedarone is the first anti-arrhythmic agent that has been demonstrated to reduce cardiovascular morbidity and mortality. Whether this will alter the overall approach of using rate vs rhythm control strategy for AF management needs to be further evaluated. Longer-term 
postmarketing studies are important, to continue to monitor potential unexpected long-term adverse events.

\section{Conclusion}

Dronedarone has been demonstrated to be effective in reducing the incidence of recurrence of $\mathrm{AF}$, although it appears to be less effective but better tolerated than amiodarone. Dronedarone appears to have a low proarrhythmic risk and is the first anti-arrhythmic that has been demonstrated to reduce cardiovascular morbidity and mortality, in clinically stable patients with other risk factors for recurrent AF. Therefore, dronedarone can be recommended as an anti-arrhythmic of choice in clinically stable patients with associated risk factors for recurrent AF, including those with no heart disease, hypertension without left ventricular hypertrophy, or coronary artery disease. Amiodarone may be reserved as an alternate agent in patients failing on dronedarone and where it is essential to maintain the patient in SR. However, if dronedarone is to be used in a patient with chronic stable heart failure, the patient must be monitored closely for any worsening of heart failure symptoms. The drug must be discontinued should the heart failure symptoms worsen. Because dronedarone is not indicated for acute cardioversion, it is probably unlikely that dronedarone will be initiated in an emergency department setting. However, health care professionals practicing in emergency room settings should monitor worsening of signs and symptoms of heart failure in patients presenting on dronedarone and discontinue the drug if that occurs. The most common side effects reported for dronedarone include diarrhea, nausea, and vomiting. Transient increase in serum creatinine due to dronedarone's inhibition of renal tubular secretion of creatinine has been observed in clinical trials, where it occurred early after treatment and reached a plateau after 7 days.

\section{Disclosure}

The author reports no conflicts of interest in this work.

\section{References}

1. American Heart Association. 2010 Heart Disease and Stroke Statistics (online). Available at: http://circ.ahajournals.org/cgi/reprint/ CIRCULATIONAHA.109.192667. Accessed June 8, 2011.

2. Lloyd-Jones DM, Wang TJ, Leip EP, et al. Lifetime risk for development of atrial fibrillation: the Framingham Heart Study. Circulation. 2004; 110(9):1042-1046.

3. Wann LS, Curtis AB, January CT, et al. 2011 ACCF/AHA/HRS Focused update on the management of patients with atrial fibrillation (Updating the 2006 Guideline). Circulation. 2011;123(1):104-123.

4. The Atrial Fibrillation Follow-up Investigation of Rhythm Management (AFFIRM) Investigators. A comparison of rate control and rhythm control in patients with atrial fibrillations. N Engl J Med. 2002;347(23): 1825-1833.
5. Reiffel JA. A contemporary look at classic trials in atrial fibrillation: what do they really show and how might they apply to future therapies? Am J Cardiol. 2008;102(6 A):3H-11H.

6. Nichol G, McAlister F, Pham B, et al. Meta-analysis of randomized controlled trials of the effectiveness of antiarrhythmic agents at promoting sinus rhythm in patients with atrial fibrillation. Heart. 2002; 87(6):535-543.

7. Singh BN, Singh SN, Reda DJ, et al. Amiodarone versus sotalol for atrial fibrillation. N Engl J Med. 2005;352(18):1861-1872.

8. Roy D, Talajic M, Dorian P, et al. Amiodarone to prevent recurrence of atrial fibrillation. Canadian Trial of Atrial Fibrillation Investigators. N Engl J Med. 2000;342(13):913-920.

9. Singh SN, Tang CX, Singh BN, et al. Quality of life and exercise performance in patients in sinus rhythm versus persistent atrial fibrillation. J Am Coll Cardiol. 2006;48(4):721-730.

10. The AFFIRM First Antiarrhythmic Drug Substudy Investigators. Maintenance of sinus rhythm in patients with atrial fibrillation: an AFFIRM substudy of the first antiarrhythmic drug. J Am Coll Cardiol. 2003;42(1):20-29.

11. Deedwania PK, Singh BN, Ellenbogan K, et al. Spontaneous conversion and maintenance of sinus rhythm by amiodarone in patients with heart failure and atrial fibrillation. Observations from the Veterans Affairs Congestive Heart Failure Survival Trial of Antiarrhythmic Therapy (CHF-STAT). Circulation. 1998;98(23):2574-2579.

12. Singh SN, Fletcher RD, Fisher SG, et al. Amiodarone in patients with congestive heart failure and asymptomatic ventricular arrhythmias. N Engl J Med. 1995;333(2):77-82.

13. Camm AJ. Safety considerations in the pharmacological management of atrial fibrillation. International Journal of Cardiology. 2008;127(3): 299-306.

14. Multaq ${ }^{\circledR}$ (Dronedarone) Briefing Document. Advisory Committee Meeting of the Cardiovascular and Renal Drugs Division of the US Food and Drug Administration. March 18, 2009. Available at: http:/www.fda.gov/ downloads/AdvisoryCommittees/CommitteesMeetingMaterials/Drugs/ CardiovascularandRenalDrugsAdvisoryCommittee/UCM134981.pdf. Accessed on June 11, 2011.

15. Kathöfer S, Thomas D, Karle CA. The novel antiarrhythmic drug dronedarone: comparison with amiodarone. Cardiovasc Drug Rev. 2005;23(3):217-230.

16. Patel PD, Bhuriya R, Patel DD, Arora BL, Singh PP, Arora RR. Dronedarone for atrial fibrillation: a new therapeutic agent. Vasc Health Risk Manag. 2009;5:635-642.

17. Sun W, Sarma JS, Singh BN. Chronic and acute effects of dronedarone on the action potential of rabbit atrial muscle preparations: comparison with amiodarone. J Cardiovasc Pharmacol. 2002;39(5):677-684.

18. Thomas D, Kathofer S, Zhang W, et al. Acute effects of dronedarone on both components of the cardiac delayed rectifier $\mathrm{K}^{+}$current, HERG and KvLQT1/minK potassium channels. Br J Pharmacol. 2003;140(5): 996-1002.

19. Gautier P, Guillemare E, Marion A, Bertrand JP, Tourneur Y, Nisato D. Electrophysiologic characterization of dronedarone in guinea pig ventricular cells. J Cardiovasc Pharmacol. 2003;41(2):191-202.

20. Hodeige D, Heyndrickx JP, Chatelain P, Manning A. SR 33589, a new amiodarone-like antiarrhythmic agent: anti-adrenoceptor activity in anaesthetized and conscious dogs. Eur J Pharmacol. 1995;279(1):25-32.

21. Multaq ${ }^{\circledR}$ Prescribing Information. Available at: http://www.multaq. com/docs/consumer_pdf/pi.aspx. Accessed on June 1, 2011.

22. Damy T, Pousset F, Caplain H, Hulot JS, Lechat P. Pharmacokinetics and pharmacodynamics interactions between metoprolol and dronedarone in extensive and poor CYP2D6 metabolizers healthy subjects. Fundam Clin Pharmacol. 2004;18(1):113-123.

23. Touboul P, Brugada J, Capucci A, Crijns HJ, Edvardsson N, Hohnloser SH. Dronedarone for prevention of atrial fibrillation: a dose-ranging study. Eur Heart J. 2003;24(16):1481-1487.

24. Singh BN, Connolly SJ, Crijns HJ, et al. Dronedarone for maintenance of sinus rhythm in atrial fibrillation or flutter. $N$ Engl J Med. 2007; 357(10):987-999. 
25. Davy JM, Herold M, Hoglund C, et al. Dronedarone for the control of ventricular rate in permanent atrial fibrillation: the Efficacy and safety of dronedarone for the control of ventricular rate during atrial fibrillation (ERATO) study. Am Heart J. 2008;156(3):527. e1-e9.

26. Køber L, Torp-Pedersen C, McMurray JJ, et al. Increased mortality after dronedarone therapy for severe heart failure. N Engl J Med. 2008; 358(25):2678-2687.

27. Hohnloser SH, Crijns HJ, van Eickels M, et al. Effect of dronedarone on cardiovascular events in atrial fibrillation. N Engl J Med. 2009; 360(7): $668-678$.

28. Torp-Pedersen C, Crijn HJ, Gaudin C, et al. Impact of dronedarone on hospitalization burden in patients with atrial fibrillation: results from the ATHENA study. Europace. 2011;13(8):1118-1126.

29. Page RL, Connolly SJ, Crijns JGM, et al. Rhythm and rate controlling effects of dronedarone in patients with atrial fibrillation (from the ATHENA trial). Am J Cardiol. 2011;107(7):1019-1022.

30. Hohnloser SH, Crijns HJ, van Eickels M, et al; ATHENA Investigators. Dronedarone in patients with congestive heart failure: insights from ATHENA. Eur Heart J. 2010;31(14):1717-1721.

31. Le Heuzey JY, De Ferrari GM, Eadzik D, Santini M, Zhu J, Davy JM. A short-term randomized, double-blinded, parallel-group study to evaluate the efficacy and safety of dronedarone versus amiodarone in patients with persistent atrial fibrillation: the DIONYSOS study. J Cardiovasc Electrophysiol. 2010;21(6):597-605.
32. Piccini JP, Hasselblad V, Peterson ED, Washam ED, Califf RM, Kong DF. Comparative efficacy of dronedarone and amiodarone for the maintenance of sinus rhythm in patients with atrial fibrillation. J Am Coll Cardiol. 2009;54(12):1089-1095.

33. FDA Drug Safety Communication: Severe liver injury associated with the use of dronedarone (marketed as Multaq). Available at: http://www. fda.gov/drugs/drugsafety/ucm240011.htm. Accessed on June 8, 2011.

34. Wood S. Deaths doubled with dronedarone in PALLAS: FDA and EMA updates. Available at: http://www.theheart.org/article/1255799. do. Accessed on September 1, 2011.

35. Optimal Timing of Dronedarone Initiation After Conversion in Patients With Persistent Atrial Fibrillation (ARTEMIS Load) Study. Available at: http://clinicaltrials.gov/ct2/show/NCT01140581. Accessed on September 1, 2011.

36. Freemantle N, Lafuente-Lafuente C, Mitchell S, Eckert L, Reynolds M. Mixed treatment comparison of dronedarone, amiodarone, sotalol, flecainide, and propafenone, for the management of atrial fibrillation. Europace. 2011;13(3):329-345

37. Cordarone ${ }^{\circledR}$ Prescribing Information. Available at: http://labeling.pfizer. com/showlabeling.aspx?id=93. Accessed on September 1, 2011.
Open Access Emergency Medicine

\section{Publish your work in this journal}

Open Access Emergency Medicine is an international, peer-reviewed, open access journal publishing original research, reports, editorials, reviews and commentaries on all aspects of emergency medicine. The manuscript management system is completely online and includes a very quick and fair peer-review system, which is all easy to use.

\section{Dovepress}

Visit http://www.dovepress.com/testimonials.php to read real quotes from published authors. 